\title{
Se former tout au long de la vie
}

Quelle nouveauté ? Quelles conditions?

Philippe Méhaut

\section{CpenEdition}

\section{Journals}

Édition électronique

URL : http://journals.openedition.org/ries/3040

DOI : 10.4000/ries.3040

ISSN : 2261-4265

Éditeur

Centre international d'études pédagogiques

Édition imprimée

Date de publication : 1 décembre 1997

Pagination : 79-88

ISSN : $1254-4590$

Référence électronique

Philippe Méhaut, «Se former tout au long de la vie », Revue internationale d'éducation de Sèvres [En ligne], 16 | Décembre 1997, mis en ligne le 07 juin 2013, consulté le 19 avril 2019. URL : http:// journals.openedition.org/ries/3040; DOI : 10.4000/ries.3040

Ce document a été généré automatiquement le 19 avril 2019

(c) Tous droits réservés 


\title{
Se former tout au long de la vie
}

\author{
Quelle nouveauté ? Quelles conditions?
}

\author{
Philippe Méhaut
}

1 Les diverses initiatives de l'Union européenne (Livre blanc: Enseigner et apprendre - Vers la société cognitive, Année européenne de la «formation tout au long de la vie») ont contribué à relancer le débat sur le rôle de la formation continue et des apprentissages par le travail dans la société française. Ce débat intervient par ailleurs au moment où d'intenses réflexions sont en cours sur l'évolution des dispositifs de certification ${ }^{1}$ mais aussi sur les limites de la promotion sociale à la française ${ }^{2}$.

\section{Idées neuves et questions récurrentes}

2 Par certains aspects, reparler de la formation tout au long de la vie pourrait donner l'impression d'un retour sur des questions déjà largement évoquées et débattues dans les trente dernières années.

3 Au plan international, il faut se souvenir que le conseil de l'Europe, l'Unesco puis l'OCDE ont contribué à amener sur le devant de la scène les thèmes de l'éducation permanente, puis de l'éducation $\mathrm{n}$ récurrente. D'une organisation à l'autre, l'angle d'attaque variait: priorité à une option de type égalité des chances dans une perspective humaniste pour les deux premières, accent mis sur les nouvelles conditions économiques mais aussi sur l'alternative à la prolongation des études pour la troisième ${ }^{3}$.

4 En France, l'historique réalisé par C. Dubar et al. ${ }^{4}$ retrace bien l'évolution d'un débat aux racines très anciennes. Les années 1970 sont l'occasion d'une réponse forte organisée autour de la loi de 1971. Celle-ci ambitionne de concilier des impératifs de formation des salariés (avec le versant obligation de l'entreprise) et des impératifs de deuxième chance, de promotion sociale mais aussi d'accès à la culture, entre autres avec le congé individuel de formation (CIF). Une forme de compromis social est alors trouvé, fortement marqué à l'époque par l'hypothèse d'une poursuite de la croissance économique, d'évolution technologique certes rapide mais ne remettant pas fondamentalement en cause les grandes tendances organisationnelles. Développer la formation à l'initiative de 
l'entreprise est alors le moyen d'accompagner l'évolution des besoins de qualification des salariés. Ouvrir, avec le CIF, un droit nouveau à une formation longue à l'initiative individuelle vient rajouter une opportunité aux dispositifs de promotion sociale existants.

L'initiative de l'Union européenne et son écho en France pourrait alors apparaître comme la relance d'une idée ancienne, un peu utopique et jamais réellement concrétisée de l'égalité des chances par la formation permanente. Cette relance intervient cependant dans une perspective radicalement nouvelle dans le Livre blanc communautaire et dans un contexte de changement significatif en France.

\section{Les présupposés du Livre blanc}

6 Ceux-ci peuvent être résumés par trois mots clés : société de l'information, globalisation et flexibilité.

7 Sur le premier registre, l'idée centrale est que l'avènement des nouvelles technologies de l'information va faire évoluer radicalement le rapport au savoir. D'une part, les exigences de maîtrise de ces technologies dans leur usage quotidien (travail et hors travail) vont se renforcer, alors même que leur propre rythme d'évolution s'accélérerait. La société ne pourrait alors faire autrement que suivre cet avènement d'un nouveau paradigme technologique. Mais, d'autre part, ces nouvelles technologies offriraient un espace radicalement différent aux processus d'acquisition des connaissances: auto apprentissage, réseaux d'échanges de savoir... Le primat des formes classiques de transmission des connaissances sous-tendu par l'école et la formation continue formalisée serait battu en brèche.

8 Sur le deuxième registre, l'Europe, prise dans le contexte de globalisation ne saurait rester compétitive que par le choix d'une option de systèmes de production à haute valeur ajoutée, dans lesquels la connaissance joue un rôle central. Il conviendrait donc d'élever en permanence le niveau de qualification de la force de travail. Or, l'école trouve des limites (de moyens, d'adaptation, d'exclusion de certaines catégories) que seules peuvent lever l'articulation de ce nouveau rapport au savoir et de ces nouvelles technologies.

9 Sur le troisième registre, les marchés du travail européens sont amenés à évoluer vers une plus grande flexibilité. La forme canonique - commune à la plupart des pays européens - d'une relation salariale relativement stable, inscrite dans la durée de la vie évoluerait inévitablement vers des formes plus souples, mêlant activité salariée, autres formes d'activités sociales ou économiques, auto-emploi et déportant vers l'individu la responsabilité de l'entretien permanent de sa qualification.

10 Certes, il s'agit là d'une interprétation durcie de propositions ouvertes qui ont, du point de vue communautaire, plus valeur d'alerte et de mise en débat que de propositions fermes. Mais les formes actuelles des initiatives communautaires autour de l'accréditation individuelle et du portefeuille de compétence incitent à garder à l'esprit cette interprétation ${ }^{5}$.

11 En tout état de cause, on voit combien le débat peut être radicalement déplacé par rapport à la problématique plus ancienne de la formation permanente :

- inscription des individus dans un univers beaucoup plus instable (tant du point de vue économique que social) où la fluidité sociale ne prendrait plus la forme de la mobilité ascensionnelle, avec dissociation du potentiel de la formation et du statut salarial ; 
- dépassement radical du clivage travail/hors travail, formation professionnelle/ épanouissement culturel ;

- conception renouvelée de la formation dans laquelle les modes formalisés de transmission/ acquisition des savoirs doivent se combiner avec d'autres formes (et donc évolution des espaces d'apprentissage dans le travail, la vie familiale et sociale).

\section{la force du système français de formation continue et sur son articulation à la formation} initiale.

\section{Un mythe fondateur...}

L'idéal de la promotion sociale par la formation est profondément ancré dans la tradition française. C'est particulièrement au début des années 1960 que se développe de façon plus systématique son mythe fondateur. Alors que la scolarité initiale passe progressivement de 14 à 16 ans et que s'exprime un besoin croissant en techniciens, la politique publique organise un droit à la « deuxième chance " pour ceux qui n'ont pas eu accès aux études initiales. Celui-ci repose sur l'hypothèse de la mise en correspondance de deux échelles sociales. D'un côté, l'échelle éducative sur laquelle, dans le contexte de l'époque, certains voient leurs chances limitées dès le départ. Il convient donc, dans une perspective égalitaire et de libération par la formation, d'ouvrir des opportunités permettant de compenser cette inégalité initiale. L'État, investi d'une responsabilité première, doit offrir les possibilités d'un rattrapage éducatif, à travers les cours du soir, notamment dans les établissements professionnels et les différentes opportunités de "formation de promotion sociale » (année à temps plein pour préparer un diplôme de technicien, CNAM ${ }^{6}$ ...).

La deuxième échelle est celle de la hiérarchie des emplois. Des distinctions claires de niveau y sont formalisées, notamment par les grilles de classification de type «PARODI ${ }^{7}$, mais avec une possibilité de passage de l'ouvrier à l'ingénieur. Cette gradation correspond à la hiérarchie des emplois au sein de la grande industrie. Dans un contexte de croissance de l'emploi mais aussi de pénurie relative de diplômés issus de la formation initiale, les opportunités de mobilité professionnelle ascendante, régulées par l'ancienneté et complétées éventuellement par la formation sont réelles.

La promotion sociale à la française, supposant un effort individuel long (on s'engage parfois dans un cursus de cours du soir sur plusieurs années) et un soutien public (gratuité des formations, parfois prise en charge du salarié pour les formations à temps plein) se veut un projet économique et social de mise en correspondance de ces deux échelles. Dans la pratique, il s'agit plus d'un mythe, certes fondateur et structurant, que d'une pratique sociale massive. Seule une petite minorité parvient en haut de l'échelle éducative par la voie d'une formation de promotion sociale qui s'avère aussi sélective que l'éducation initiale. Par ailleurs, nombre de trajectoires relèvent plutôt d'un rattrapage social, suite à un déclassement initial par rapport à la position du père, plutôt que d'une ascension en continuité. 


\section{... en profonde déstabilisation}

16

à l'enseignement supérieur viennent modifier profondément la donne. D'une part, les laissés pour compte sont moins nombreux. L'idée d'une démocratisation potentielle domine et il y a moins d'urgence apparente à offrir une deuxième chance à ceux qui n'ont pas pu (ou su) saisir la première. Le récent bilan établi par Goux et Maurin ${ }^{8}$ à partir de l'enquête $\mathrm{FQP}^{9}$, confirme l'idée d'une démocratisation relative, par déplacement « homothétique » de la structure de scolarisation, qui ouvre certes des opportunités plus fortes aux enfants de famille modeste, mais sans modifier radicalement leur position relative dans la hiérarchie scolaire. Mais surtout, il ouvre une perspective d'interprétation, déjà ancienne, qui recoupe certaines des interpellations de la société cognitive : les déterminants économiques qui expliquent une part de ces inégalités sont toujours à l'œuvre. Mais les déterminants «culturels » sont très forts (par exemple, la capacité des familles aisées à maîtriser les arcanes du système éducatif). Si l'on confronte un tel constat à l'hypothèse du rôle croissant des nouvelles technologies de l'information, alors on mesure combien l'enjeu est fort par rapport à des risques de remarginalisation.

L'évolution de la politique publique de formation continue va aussi contribuer au recul des formations traditionnelles de promotion sociale.

D'une part, les développements impulsés par la loi de 1971 vont, en quelque sorte, recentrer l'économie générale du système de formation continue sur l'entreprise, avec un fort développement des formations courtes, non diplomates. Certes, les probabilités d'accès à une formation continue courte sont infiniment plus élevées aujourd'hui qu'hier. Certes aussi, on peut parler d'une légère tendance à la réduction des inégalités d'accès ${ }^{10}$. Mais, là encore, comme le soulignent Goux et Maurin, le « rendement » individuel de ce type de formation en termes de salaire ou de carrière est faible. L'effet principal est celui de la stabilisation dans l'entreprise. Or, si l'on reprend l'hypothèse de la flexibilité croissante et d'une évolution plus ou moins radicale de la relation salariale, c'est vers ceux qui sont les plus mobiles (mobilité contrainte ou non), vers les interstices entre deux emplois que se déplace l'enjeu de la formation "permanente ». De ce point de vue, en dehors du traitement social du chômage, les dispositifs français apparaissent assez démunis. En effet, la focalisation croissante de l'intervention publique sur la formation d'insertion des jeunes et sur la formation des chômeurs s'est opérée au détriment du soutien aux formations de promotion sociale plus traditionnelles ou de formes renouvelées.

Du côté de l'échelle des emplois et des positions sociales acquises dans le travail, les mouvements sont tout aussi forts. Rappelons-en quelques composantes. D'abord la crise de l'emploi concerne au premier chef la grande industrie, la plus porteuse du modèle de la promotion sociale. Cette crise réduit mathématiquement les opportunités de promotion. Le blocage de l'embauche conduit par ailleurs à des structures démographiques vieillissantes qui ne sont pas particulièrement porteuses du point de vue de la promotion. Et si la mobilité interentreprises a repris, celle-ci s'accompagne souvent du passage par le chômage et s'inscrit moins dans un horizon promotionnel à moyen 
terme. De plus, on sait que ceux qui changent d'entreprise sont aussi ceux qui ont le moins accès à la formation continue.

Il faudrait ajouter à ce diagnostic les transformations dans les modes de vie et de travail. A première vue, la réduction du temps de travail ouvre un espace nouveau à la formation. Toutefois, cette évolution est contrecarrée par deux aspects. D'une part, la montée des temps contraints (notamment temps de déplacement) qui vient limiter sensiblement le gain laissé par la baisse du temps de travail. D'autre part, la croissance significative de l'emploi et du taux d'activité féminin qui, on le sait, ne s'accompagne pas d'une transformation aussi rapide des modes de vie et qui laisse le temps féminin «non contraint » significativement en dessous du temps masculin.

\section{Une forte demande latente}

En dépit de cette déstabilisation, la demande individuelle de formation continue longue, répondant à des usages sociaux variés ne se dément pas. Mais il faut alors quitter les catégories de la promotion sociale "traditionnelle», au sens de la mise en correspondance des deux échelles ci-dessus pour porter un diagnostic plus fin.

Qu'il s'agisse du CNAM, du CESI ${ }^{11}$, des cours assurés par les municipalités, des associations philotechniques ou d'éducation populaire, les structures dont la vocation initiale était la plus proche de la promotion sociale connaissent un maintien voire une croissance de leurs effectifs de stagiaires, mais avec un public plus diversifié dans ses statuts et ses attentes. Il en va de même du côté des GRETA où l'on recense 35000 stagiaires individuels des centres de formation du ministère de l'Agriculture. C'est probablement l'université qui, dans la dernière décennie, a dû affronter la croissance d'une demande nouvelle en provenance d'adultes reprenant le chemin des études. On estime en effet à près de 160000 les inscriptions annuelles d'adultes en reprise d'études. On pourrait enfin ajouter à cette liste une fraction du public de l'enseignement à distance, mais aussi des stagiaires qui suivent une formation longue, souvent diplomate à l'initiative de leur entreprise.

Toutefois, la difficulté à redéfinir et à mesurer l'ensemble des formations « de promotion sociale » correspond bien au brouillage évoqué sur les deux échelles. Sur la première, les attentes sont plus diversifiées : recherche d'un diplôme plus élevé dans une formation voisine de sa formation ou de son métier d'origine, tentative de reconversion, recherche d'un diplôme complémentaire dans une autre spécialité, recherche d'une formation validée mais non nécessairement diplomate à visée professionnelle, ou recherche d'une formation d'ouverture, de culture, non immédiatement liée à un projet professionnel. L'éventail des motivations semble s'être singulièrement élargi. Il en va de même sur la deuxième échelle. Certains projets et certaines trajectoires gardent les caractéristiques de mise en correspondance des deux échelles : relative linéarité du parcours de formation et du parcours d'emploi (par exemple, passage de technicien à ingénieur). D'autres témoignent d'une reconversion professionnelle qui ne peut pas toujours s'analyser en terme promotionnel. D'autres, plus chaotiques, expriment les difficultés à construire un parcours dans un contexte d'emploi difficile et font de la formation un moyen d'échapper au chômage, par exemple, à la suite d'un licenciement. D'autres sont inscrites dans l'allongement de l'accès à l'emploi pour les jeunes ; la formation « continue » est alors un prolongement d'une trajectoire de formation initiale, plutôt dans une logique de poursuite d'études différée. 
La question de la formation tout au long de la vie s'exprime alors bien dans un contexte économique et social différent et interpelle les conceptions anciennes de la formation c continue et plus particulièrement des formations de promotion sociale. Il faut alors s'interroger sur les conditions permissives d' un déplacement de problématique mais aussi sur les nécessités politiques indispensables pour donner vie à la formation tout au long de la vie.

\section{Quelles opportunités, quelles conditions ?}

Du côté des systèmes de travail, l'évolution engagée semble ouvrir plus de possibilités pour une autre conception de la relatent travail, formation et apprentissage. Dans certaines sphères de l'industrie et des services, le recul relatif des formes les plus tayloriennes ouvre des espaces (temporels et organisationnels) à une meilleure acquisition des connaissances. Le développement des démarches de certification qualité va par exemple dans ce sens. Nous avons pu parler, sur la base de l'examen de diverses innovations organisationnelles en Europe, d'un double processus de mise en reconnaissance: mise en reconnaissance de savoirs et savoir-faire existants potentiellement, mais niés par les formes d'organisation du travail antérieures; mise en reconnaissance de savoir " externes " portés par les individus mais, là encore, niés dans les formes anciennes d'organisation du travail. Cette mise en reconnaissance, dans le cadre d'organisations qui sollicitent plus l'initiative et la polyvalence, contribue à réintroduire dans le travail et son organisation des préoccupations en termes d'apprentissage qui en avaient été « expulsées » à la fois par la tendance à la taylorisation, mais aussi par le retrait progressif des entreprises de leurs responsabilités formatrices ${ }^{12}$.

Ceci est vrai aussi des espaces «nouveaux » d'emploi, où la dimension relationnelle est souvent forte, qui mettent l'accent sur les « introuvables» compétences transversales. Ces opportunités nouvelles vont souvent de pair avec l'usage accru des nouvelles technologies de l'information dans et hors le travail. Mais il faut voir aussi combien elles peuvent être porteuses d'exclusion, dès lors que le socle des pré requis n'est pas garanti et dès lors qu'il apparait plus facile, dans un contexte de fort chômage et de sorties à des niveaux élevés du système éducatif de privilégier des recrutements externes et des logiques de substitution.

Par ailleurs, dans une conception très libérale de la flexibilité, où l'on privilégierait la voie d'une flexibilité systématique des contrats de travail et où l'on reporterait sur l'individu la charge de la preuve, c'est-à-dire de sa formation permanente, on peut craindre que s'amplifient les constats déjà existants : formation continue et stabilité dans l'emploi vont de pair (c'est aux plus stables que va la formation ou la formation stabilise le noyau dur), formation initiale et formation continue vont de pair (l'accès à la formation continue est plus ouvert à ceux qui sont les mieux formés). Les espaces de l'emploi porteurs d'un potentiel de professionnalisation pour les moins formés sont aussi ceux qui peuvent être le plus soumis à des logiques marchandes, à la précarité, avec ce qu'elle signifie de difficultés d'accès à la formation formalisée, comme de difficultés à construire des parcours d'acquisition de la qualification par le travail.

Parler de formation tout au long de la vie, c'est donc se situer dans une évolution des systèmes de travail et d'emploi qui privilégie des logiques de professionnalisation individuelles et collectives. Incitation publique et construction de règles collectives sont 
alors une nécessité pour éviter que les espaces nouveaux de l'emploi soient ceux où la notion de formation tout au long de la vie prenne le moins de sens.

Du côté des systèmes de formation, il faut probablement prendre toute la mesure des acquis du système français. D'un côté, une reconnaissance forte par la puissance publique d'un droit à l'éducation et à la formation professionnelle, sous-tendu aujourd'hui par une politique volontariste dans le champ de la formation initiale et par des dispositifs limités mais originaux dans celui de la formation continue. De l'autre, un compromis social autour de la loi de 1971 qui a puissamment contribué à ancrer l'idée de formation continue dans la société et à infléchir des comportements d'entreprise en réintroduisant la préoccupation formation au sein de cette dernière. Mais les faiblesses actuelles sont au moins de trois ordres :

- la croissance de la scolarisation initiale peut déstabiliser l'idée de formation continue : la deuxième chance apparaît plus difficile ; mieux vaut prolonger au maximum ses études que de miser sur un retour ultérieur en formation;

- ce, d'autant plus que cette croissance renforce quasi mathématiquement le poids du modèle scolaire : la reconnaissance des acquis par le travail, les formes alternatives de formation ont d'autant moins d'espace ;

- enfin, la dichotomie croissante entre ce qui relève de l'entreprise dans le champ de la formation continue et ce qui relève d'une initiative individuelle qui ne trouve plus à se satisfaire dans les dispositifs classiques, révèle l'épuisement du compromis social des années $1970^{13}$.

31 Trois conditions sont alors à remplir. La première est incontestablement celle d'un rééquilibrage du système français de formation continue entre ce qui relève de l'initiative de l'entreprise et de celle de l'individu. Mais entre une voie «à l'anglaise ", où la charge de la preuve repose essentiellement sur l'individu et une voie plus contractuelle où État et partenaires s'engageraient à assurer une continuité de statut et à ouvrir, par l'évolution du temps de travail ou la construction d'un statut d'activité incluant travail et formation, des espaces nouveaux à cette initiative individuelle, l'éventail des possibles est large. Du congé individuel de formation au capital temps, des dispositifs existent. Leur usage reste cependant très limité. Par ailleurs, dès lors que l'on accepte l'hypothèse d'une mobilité professionnelle croissante, le plus souvent avec changement d'entreprise, c'est l'outillage statutaire de ce parcours de mobilité, dont la formation est une étape, qui est en cause, si l'on veut éviter que la formation continue soit en quelque sorte prise en tenaille par le chômage : d'un côté, rejetée par crainte de la perte d'emploi ou, de l'autre, contrainte par le chômage.

32 La deuxième réside dans une meilleure articulation entre formation initiale et formation continue. Ceci concerne d'une part la reconnaissance effective d'un droit positif à organiser son parcours de formation en choisissant entre poursuite d'études ou retour en formation ultérieurement. On pourrait, par exemple, envisager que le capital temps formation soit bonifié pour ceux qui arrêtent leurs études avant l'entrée dans l'enseignement supérieur. Mais on pourrait aussi considérer que certaines structures de formation initiale soient plus résolument organisées pour faciliter la présence d'adultes, dans et hors le temps de travail (modularisation, double année universitaire...).

La troisième touche aux dispositifs de certification, dans la mesure où le diplôme dans sa conception actuelle, contribue à normer fortement pédagogie et cursus de formation continue sur le modèle de la formation initiale. Ouvrir plus largement les voies de la validation des acquis et de formes alternatives de certification pourrait contribuer à 
distancier la formation initiale d'une formation continue qui s'adresse à des publics mieux formés qu'il y a vingt ans et qui, ainsi, retrouveraient un espace d'autonomie et de redéploiement. Une telle évolution aurait probablement un impact significatif sur la pédagogie et sur les possibilités de développement des nouvelles technologies éducatives.

\section{BIBLIOGRAPHIE}

CORREIA M., "Formation et promotion sociale, des liens qui se distendent », Actualité de la formation permanente, $\mathrm{n}^{\circ} 141,1996, \mathrm{p} .48-57$.

DUBAR C, « Pour une approche compréhensive de la promotion sociale », Pour, n 148, 1995, p. $39-49$.

DUBAR C, PODEVIN G., « Formation et promotion en France depuis 20 ans », Céreq, Bref, n 59, $1990,4 \mathrm{p}$.

FOND HARMAND L., « Approche biographique et retour aux études », Éducation Permanente, $\mathrm{n}^{\circ}$ 125,1995, p. 7-25.

KALCK P., GAUTIER F., Du technicien au cadre : le rôle des formations continues diplômantes, Céreq, Document $n^{\circ}$ 109, 1995.

PODEVIN G., « De la promotion sociale à la promotion de l'économique », Pour, nº 14, 1995, p. 65-67.

TERROT N., Histoire de l'éducation des adultes en France, Édilig, 1983.

\section{NOTES}

1. Cf. le rapport De Virville, Donner un nouvel élan à la formation professionnelle, Paris, Documentation française, 1996, $148 \mathrm{p}$.

2. CNAM, Céreq, DFP, De la promotion sociale à la formation tout au long de la vie, Actes du colloque de 1996, Céreq, 1996.

3. D. Kallen, «Éducation permanente, une rétrospective », Formation professionnelle, $\mathrm{n}^{\circ} 8 / 9$, mai-décembre 1996, p. 16-22.

4. C. Dubar et alii, Les évolutions de la promotion sociale en France, essai de sociologie historique, Laboratoire Printemps, Université de Versailles-Saint-Quentin-en-Yvelines, 1996.

5. A. d'Iribarne, «Une lecture des paradigmes du Livre blanc sur l'éducation et la formation: éléments pour un débat ", Formation professionnelle, mai-décembre 1996, p. 23-32.

6. Conservatoire national des arts et métiers.

7. Grille de classement des postes (OS/OP/tecniciens...) établie dans les années 1950.

8. D. Goux, E. Maurin, « Destinées sociales : le rôle de l'école et du milieu d'origine », Économie et Statistique, $\mathrm{n}^{\circ} 306,1997$, p. 13-26.

9. Formation/qualification professionnelle. 
10. F. Aventur, S. Hanchane, Justice sociale et formation continue dans l'entreprise, rapport de recherche pour le CGP, ronéotypé, Céreq, 1997 ; D. Goux, E. Maurin, « Les entreprises, les salariés, et la formation continue ", Économie et Statistique, n 306, 1997, p. 41-56.

11. Centre d'études supérieures industrielles.

12. P. Méhaut, J. Delcourt, Le rôle de l'entreprise dans la production des qualifications: effets formateurs de l'organisation du travail, Berlin, Cedefop, 1996 (Cedefop: Centre européen pour le développement de la formation professionnelle).

13. V. Merle, «Formation professionnelle un nouveau compromis social à construire, de l'utopie à la crise », Éducation Permanente, nº 129, 1996, p. 63-74.

\section{RÉSUMÉS}

Le Livre blanc alimente en France le débat sur le rôle de la formation continue et des apprentissages par le travail. Le concept de formation tout au long de la vie ne peut avoir d'impact positif sur le devenir de l'individu et de l'entreprise si ne sont levées des contraintes fortes du système français. Après avoir passé en revue les évolutions depuis les années soixante, l'auteur identifie les conditions à remplir pour que la formation tout au long de la vie ne serve pas d'alibi dans une société en mutation. Il conclut sur l'acquis social que représente la validation, par le diplôme, de l'expérience professionnelle.

\section{INDEX}

Index géographique : France, Europe

Mots-clés : formation tout au long de la vie, formation continue, formation professionnelle

\section{AUTEUR}

\section{PHILIPPE MÉHAUT}

Directeur-adjoint, Centre de recherches sur les qualifications et les coûts(CEREQ), Marseille, France 